(7) $\int \mathrm{Q}_{n} \mathrm{~V}_{n} d \mathrm{~S}=\mathrm{MV}_{n}^{\prime}$, where $\mathrm{V}_{n}^{\prime}$ is the value of $\mathrm{V}_{n}$ at the pole of $Q$.

The surface value of the above symmetrical harmonic is

$$
\int_{0}^{\pi}\left(x+\sqrt{x^{2}-1} \cos \theta\right)^{n} \sin ^{p-3} \theta d \theta .
$$

We may consider this as a function of the single variable $x$, and it is easy from the above results to show that

$$
\int_{-1}^{+1} Q_{n} Q_{m}\left(1-x^{2}\right)^{p-3} \stackrel{2}{*} d x=0
$$

when $m, n$ are different, but $=$ a certain finite numerical quantity, casily found, when $m=n$.

The functions we have arrived at include, of course, as particular cases, simple and zonal Harmonic Functions. They are discussed at the end of Vol. I. of Heine's Kugelfunctionen.

The method of this paper is not mentioned in Heine, but $I$ have found since $I$ had it worked out that it is not new. In Professor Cayley's collected Works, Vol. I., page 397, will be found a short paper in which he proves (6) and (7). His proofs, however, are quite different from those $I$ had arrived at, and have given above.

\title{
A Method of Teaching Electrostatics in School.
}

By J. T. Morrison, M.A., B.Sc.

The object of the paper was to suggest for the teaching of electrostatics a leading idea, which should readily co-ordinate all the facts, introduce no misleading inferences, and guide the course of learners in the direction of the most recent investigations-in all which respects the notion of attraction and repulsion is at least a partial failure. The leading idea or fact referred to is, that almost all electrostatic distributions, however complex, can be analysed into one or more repetitions of a certain simple system, which is called in the paper "an electrostatic system," and which may be described as follows:-Two equally and oppositely electrified conducting surfaces, facing each other, separated by any dielectric, and insulated from each other. A complete study of one zystem of this kind, and 
of the very simple ways in which the establishment of one such system often necessitates the establishment of others, is therefore the fundamental study of electrostatics.

The following order may be adopted :-

After getting pupils to discover that there are two kinds of electrification, and to find out the difference between conductors and non-conductors, the investigation of an "electrostatic system" is begun.

A. Electric Quantity and Distribution in " an electrostatic" oystem.

Chief Result:-The surfaces are equally and oppositely electrified, and to each small part of one surface may be shown to correspond a small equally but oppositely electrified part of the other surface.

An important subsidiary principle may here be introduced -namely, the constancy of the quantity of electritication in a set of bodies that do not communicate with bodies that are outside the set. With the help of this principle, various distributions should be analysed into their constituent "electrostatic systems," and four important cases were so analysed in the paper.

B. Mass-Motions in an "electrostatic system."

Chief Result (a):-There is a mass-acceleration of each part of the two surfaces, the direction of the mass-acceleration being perpendicular to the surface.

Definition of unit of electrification.

(b) Mass acceleration on surface carrying unit positive selectrification $=-4 \pi \sigma$.

C. Energy and Potential Differcnce in an "electrostatic system."

Definition of Potential. Equipotential Surfaces.

Chief Result :-Energy $=\frac{1}{2}$ Quantity $\times$ Potential Difference.

Seat and Nature of Electrostatic Energy.

Capacity, and Specific Inductive Capacity.

D. Electric Discharge of a System.

Electric Strength.

Electric Oscillations. Transformations undergone by the Electric Energy. Electrical Radiation. 
There is no room to indicate the experimental means by which the method may be executed, nor to show how the various instruments may be introduced, their laws discovered, and constants calculated ; but these matters will be fairly obvious.

Eighth Meeting, 13th June 1890.

R. E. Allardice, Esq., M.A., Vice-President, in the Chair.

Note on the Orthomorphio Transformation of a cirole into itself.

From a letter by Professor CAYLEY.

"The following is, of course, substantially well-known, but it strikes me as rather pretty :- to find the Orthomorphic transformation of the circle

into itself. Assume this to be

$$
x^{2}+y^{2}-1=0
$$

$$
x_{1}+\iota y_{1} \frac{\mathrm{A}(x+\iota y)+\mathrm{B}}{1+\mathrm{C}(x+\imath y)} \text {. }
$$

Then, writing $A^{\prime}, B^{\prime}, O^{\prime}$ for the conjugates of $A, B, C$, we have

and then

$$
x_{1}-\iota y=\frac{A^{\prime}(x-\iota y)+B^{\prime}}{1+C^{\prime}(x-\imath y)}
$$

$$
x_{1}^{2}+y_{1}^{2}=\frac{\mathrm{AA}^{\prime}\left(x^{2}+y^{2}\right)+\mathrm{AB}^{\prime}(x+\iota y)+\mathrm{A}^{\prime} \mathrm{B}(x-\iota y)+\mathrm{BB}^{\prime}}{1+\mathrm{C}(x+\iota y)+\mathrm{C}^{\prime}(x-\iota y)+\mathrm{CC}^{\prime}\left(x^{2}+y^{2}\right)},
$$

which should be an identity for $x^{2}+y^{2}=1, x_{1}^{2}+y_{1}^{2}=1$.

Evidently $C=A B^{\prime}$, whence $C^{\prime}=A^{\prime} B$, and the equation then is

$$
1+\mathrm{AA}^{\prime} \mathrm{BB}^{\prime}=\mathrm{AA}^{\prime}+\mathrm{BB}^{\prime},
$$

i.e.,

$$
\left(1-\mathrm{AA}^{\prime}\right)\left(1-\mathrm{BB}^{\prime}\right)=0 \text {. }
$$

But $\mathrm{BB}^{\prime}=1$ gives the illusory result

therefore

$$
\begin{gathered}
x_{1}+t y_{1}=\mathbf{B}, \\
1-\mathbf{A A}^{\prime}=0,
\end{gathered}
$$

and the required solution thus is

$$
x_{1}+\iota y_{1}=\frac{\mathbf{A}(x+\iota y)+\mathbf{B}}{1+\mathbf{A B}^{\prime}(x+\iota y)}
$$

